\title{
Pineal parenchymal tumor of intermediate differentiation: Treatment outcomes of five cases
}

\author{
TSUBASA WATANABE ${ }^{1}$, TAKASHI MIZOWAKI ${ }^{1}$, YOSHIKI ARAKAWA ${ }^{2}$, YUSUKE IIZUKA ${ }^{1}$, KENGO OGURA ${ }^{1}$, \\ KATSUYUKI SAKANAKA ${ }^{1}$, SUSUMU MIYAMOTO $^{2}$ and MASAHIRO HIRAOKA ${ }^{1}$ \\ Departments of ${ }^{1}$ Radiation Oncology and Image-Applied Therapy, and ${ }^{2}$ Neurosurgery, \\ Kyoto University Graduate School of Medicine, Shogoin, Sakyo-ku, Kyoto 606-8507, Japan
}

Received May 7, 2013; Accepted December 12, 2013

DOI: $10.3892 / \mathrm{mco} .2013 .231$

\begin{abstract}
Pineal parenchymal tumor of intermediate differentiation (PPTID) is a rare disease, first classified by the World Health Organization in 2000. The number of available studies on the treatment of PPTID is currrently limited and the optimal management for this disease has not yet been determined. We retrospectively evaluated the treatment outcomes for PPTID at our institute and analyzed the roles of radiation therapy and chemotherapy for this disease. The clinical data on five patients diagnosed with PPTID since 2000 were retrospectively reviewed. Patients with cerebrospinal dissemination at diagnosis received biopsy-only surgery, craniospinal and whole-ventricular irradiation and chemotherapy. Patients with locally limited disease at diagnosis received local or whole-ventricular irradiation after surgery. The median relapse-free and overall survival were 72.9 and 94.1 months, respectively. Two of the five patients developed a relapse of cerebrospinal dissemination after treatment and succumbed to the disease. All the patients who received both craniospinal and whole-ventricular irradiation exhibited evidence of cerebral white matter abnormalities in magnetic resonance imaging and developed neurocognitive disorders after treatment. Although PPTID may be aggressive and has cerebrospinal fluid seeding potential, PPTID patients may survive long-term, even after recurrence. Considering the long survival time and the late adverse effects due to intensive treatment, the irradiation field and usage of chemotherapy after surgery require optimization.
\end{abstract}

Correspondence to: Dr Takashi Mizowaki, Department of Radiation Oncology and Image-Applied Therapy, Kyoto University Graduate School of Medicine, 54 Kawaharacho, Shogoin, Sakyo-ku, Kyoto 606-8507, Japan

E-mail: mizo@kuhp.kyoto-u.ac.jp

Key words: chemotherapy, pineal parenchymal tumor, pineal parenchymal tumor of intermediate differentiation, radiation therapy, surgery

\section{Introduction}

Pineal parenchymal tumors are rare, accounting for $<0.3 \%$ of all primary central nervous system tumors (1). Pineal parenchymal tumors are heterogeneous entities, exhibiting considerable morphological variation. According to the WHO classification for tumors of the central nervous system (2007 revision), these tumors are histologically subdivided into pineocytoma (grade I), pineoblastoma (grade IV), papillary tumor of the pineal region (grade II or III) and pineal parenchymal tumor of intermediate differentiation (PPTID) (grade II or III) $(2,3)$. PPTID is a fairly recently defined disease. It was first classified by the WHO in 2000 as a pineal parenchymal tumor with an intermediate prognosis between pineocytoma and pineoblastoma. Determination of the mitotic index and immunohistochemistry are used to pathologically classify PPTIDs as grade II or III $(2,3)$.

The treatment for pineal parenchymal tumors is histology-dependent. Pineocytomas are treated with surgical resection. If complete or subtotal resection is accomplished, the outcome is favorable, even without adjuvant treatment $(4,5)$. By contrast, the treatment of pineoblastomas should consist of surgery as well as adjuvant treatment, including chemotherapy and craniospinal irradiation. Occasionally, even more aggressive treatment, including myeloablative chemotherapy with stem cell rescue, may be required for the treatment of pineoblastomas (6).

Unlike other histological subtypes, the optimal management for PPTIDs remains to be determined, as it was relatively recently characterized and is rather rare. In one series, patients with PPTID who were treated with surgery alone survived free of disease (7). At the other extreme, another study described PPTIDs as tumors with seeding potential and recommended postoperative treatment in a manner similar to that for pineoblastomas (1). To the best of our knowledge, the number of available studies on the treatment of PPTID is limited, particularly regarding radiation therapy and chemotherapy (8-10), whereas there are currently no treatment reports for PPTID cases alone. The aim of this study was to retrospectively evaluate the treatment outcomes of patients with PPTID who received treatment at our institute and analyze the effects of radiation therapy and chemotherapy on this disease. 


\section{Materials and methods}

Record review. We conducted a retrospective record review for patients who were diagnosed with PPTID and treated with radiation therapy at our institute from 2000 onwards. Clinical data, including Eastern Cooperative Oncology Group performance status and Karnofsky performance status, pathological results, imaging [computed tomography (CT) and magnetic resonance imaging (MRI)], initial treatment, resection status, details of chemotherapy, details of radiation therapy, initial response to treatment, recurrence pattern and late adverse toxicities, were collected.

The extent of surgical resection was assessed according to the surgical and pathological reports and postoperative imaging. The resection status was classified as previously described: 'gross total resection' was defined as no evidence of contrast-enhancing tumor on postoperative images; 'R2-resection' was any surgical tumor resection less than gross total resection; and 'biopsy only' was no surgical tumor resection due to inoperability, with a biopsy performed to determine tumor histology (10). The response to treatment was assessed based on serial measurements of CT or MRI scans.

The criteria for response were as follows: complete response (CR), disappearance of tumor; partial response (PR), $>50 \%$ decrease in tumor size; progressive disease, $>25 \%$ increase in tumor size or any appearance of new sites; stable disease, all other situations. Relapse-free and overall survival were calculated from the initiation of radiation therapy to the date of relapse and to the date of death or last follow-up, respectively. The endpoint of the analysis was the date of either the last follow-up or the patient's death.

The appearance or exacerbation of cerebral white matter abnormalities in MRI after treatment, neurocognitive effects, pituitary function and incidence of cerebrovascular disease were investigated as late effects of treatment, according to the grading system of Radiation Therapy Oncology Group (RTOG) common toxicity criteria and Common Terminology Criteria for Adverse Events (CTCAE), version 3.0. The details of the classification according to neurocognitive disorders are as follows: RTOG (neurological/cortical) grade 1, mild somnolence or agitation; grade 2, moderate somnolence or agitation; grade 3 , severe somnolence, agitation, confusion, disorientation or hallucinations; and grade 4, coma, seizures and toxic paralysis; and CTCAE (cognitive disturbance) grade 1, mild cognitive disability, not interfering with work/school/life performance, specialized educational services/devices not indicated; grade 2, moderate cognitive disability, interfering with work/school/life performance but capable of independent living, specialized resources indicated on a part-time basis; grade 3 , severe cognitive disability, with significant impairment of work/school/life performance; and grade 4 , unable to perform activities of daily living, with full-time specialized resources or institutionalization indicated.

Patients. Five patients were diagnosed with PPTID and treated at our institute between 2000 and 2011. Table I summarizes the patients' backgrounds, tumor pathological characteristics and treatment received. Two patients were male and three were female. The median age at diagnosis was 52 years (range, $30-55$ years). Two patients had evidence of cerebrospinal dissemination at diagnosis, as assessed by radiological findings or cerebrospinal fluid (CSF) cytology. A cytological examination of the CSF was performed prior to chemotherapy and radiation therapy and craniospinal imaging was performed throughout the therapy. The grade of the disease was determined according to the number of mitoses and immunostaining for neurofilaments (grade 2, $<6$ mitoses and strongly immunopositive for neurofilaments; grade 3, $>6$ mitoses or $<6$ mitoses, but without strong immunostaining for neurofilaments) (2). The five patients were pathologically classified as WHO grade 2 according to pathological criteria (Table I).

Treatment. None of the patients underwent a gross total resection, due to the difficulty of tumor location and invasion toward the thalamus or the surrounding eloquent area. Three of the five patients had an R2-resection and the remaining two patients, who had cerebrospinal dissemination at diagnosis, had biopsy-only surgery (Table I).

All the patients were treated with external beam radiation therapy with a 1.8-Gy fraction dose after surgery. Among the five patients, two received 54 Gy directed at the primary lesion following R2-resection; the two patients with cerebrospinal dissemination at diagnosis received $36 \mathrm{~Gy}$ of craniospinal irradiation and 18 Gy of whole-ventricular irradiation after biopsy-only surgery. The remaining patient was initially planned to receive 59.4 Gy of whole-ventricular irradiation with concurrent chemotherapy following R2-resection; however, irradiation was discontinued after administering 34.2 Gy due to severe sepsis after chemotherapy.

Four of the patients received six courses of combination chemotherapy with vincristine $\left(0.6 \mathrm{mg} / \mathrm{m}^{2}\right)$, nimustine $\left(60 \mathrm{mg} / \mathrm{m}^{2}\right)$, carboplatin $\left(110 \mathrm{mg} / \mathrm{m}^{2}\right)$ and interferon $\beta$ $\left(3 \times 10^{6} \mathrm{IU}\right)$ on day 1 and the same dose of vincristine and interferon $\beta$ on days 8 and 15 (Table I). The remaining patient was administered radiation therapy alone following R2-resection. The two patients with cerebrospinal dissemination at diagnosis received sequential chemotherapy after radiation therapy and the two patients without dissemination received concurrent chemotherapy with radiation therapy after surgery.

All five patients underwent follow-up MRI or CT every 2 months during the first year after treatment. Further MRI or CT follow-up were performed every 4-6 months after the first year of follow-up.

\section{Results}

Outcome. Table II summarizes the treatment results of the five PPTID patients included in the present study. The patients appeared to have long-term survival. The median relapse-free and overall survival were 72.9 and 94.1 months, respectively (mean relapse-free survival, 65.1 months; range, 13.9-108.6 months; and mean overall survival, 79.9 months; range, 13.9-108.6 months). Three of the five patients achieved a CR and two attained a PR as an initial response to treatment (Table II). Although two of the patients developed a recurrence after treatment, they survived long-term after treatment, with overall survival times of 106.1 and 76.8 months (Table II). These results demonstrated that, with optimized surgery, radiation therapy and chemotherapy, PPTID patients have the potential for long-term survival. 

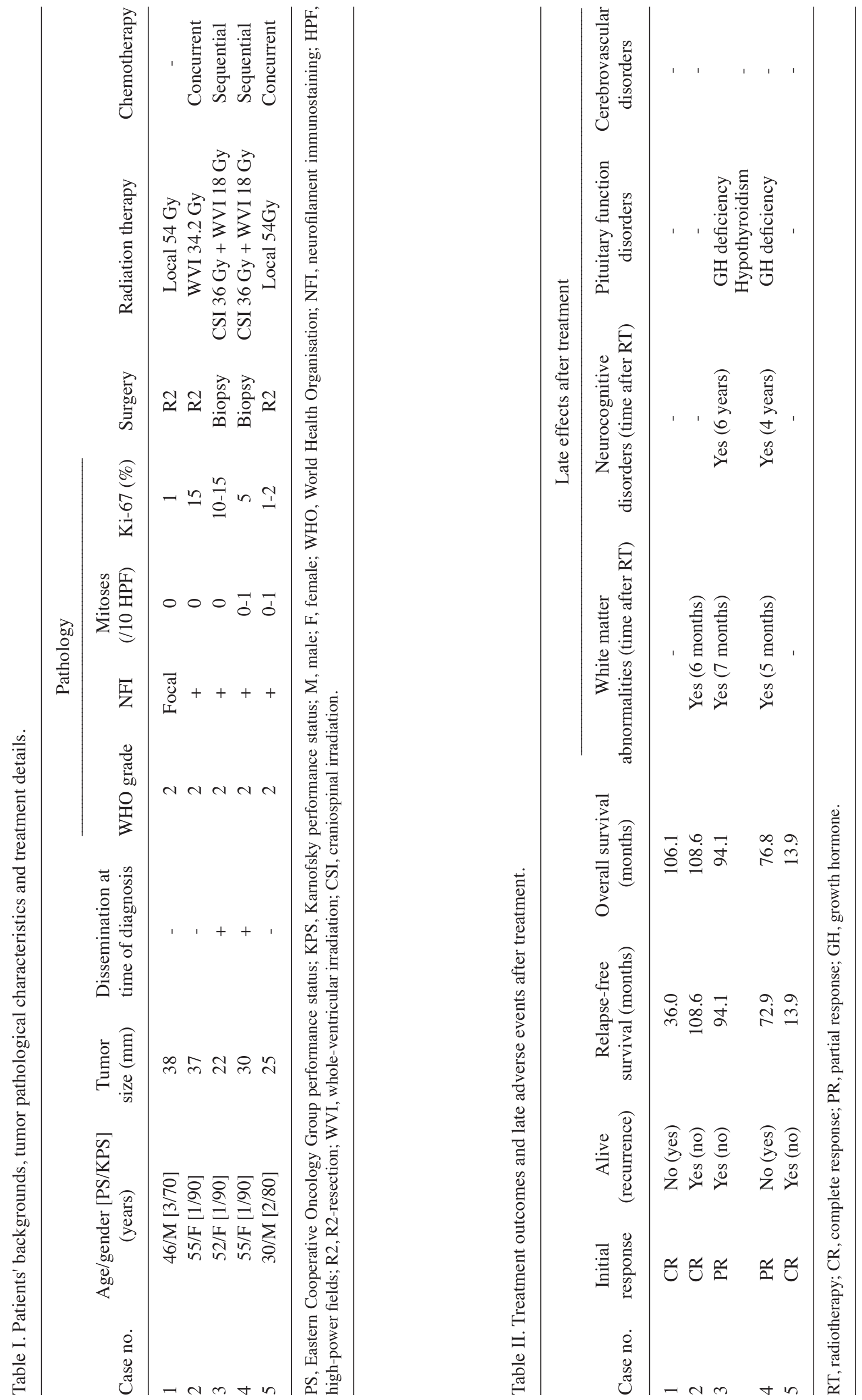


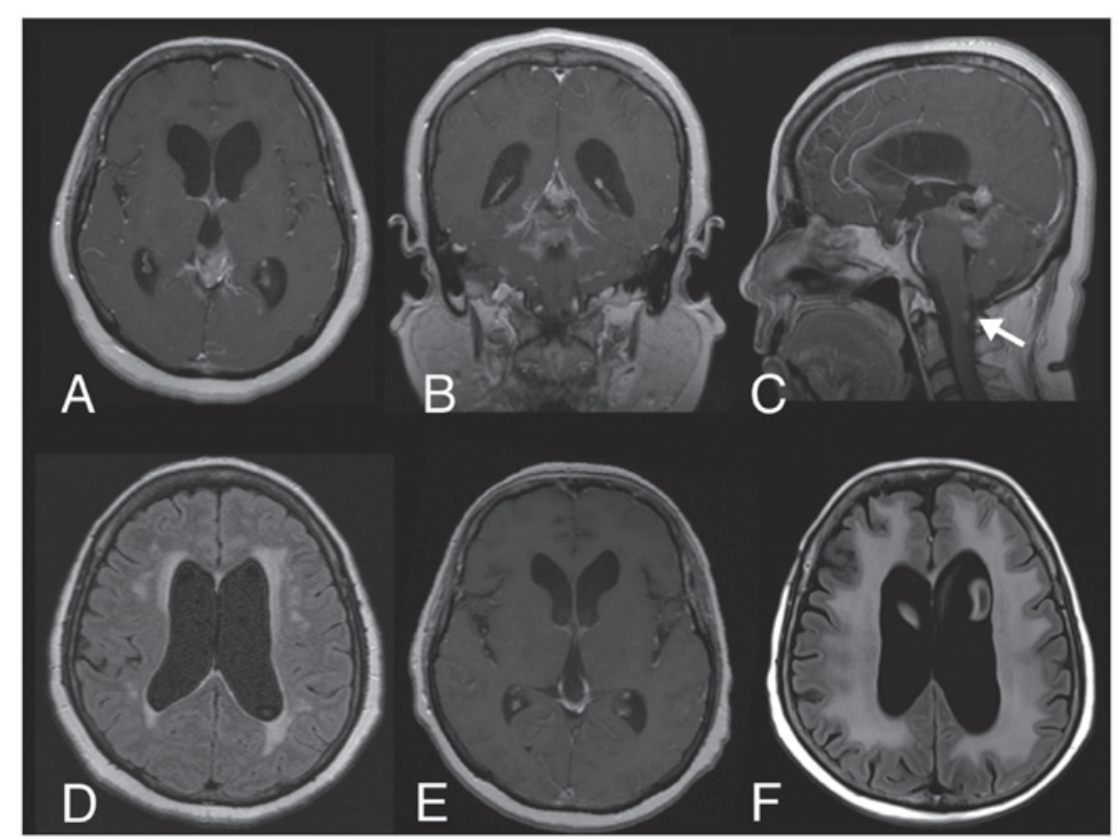

Figure 1. Example of treatment response and a late adverse effect. (A-D) Magnetic resonance imaging at the time of diagnosis (Tables I and II, case 3). (A, B and C) Contrast-enhanced T1-weighted imaging prior to treatment revealed spinal dissemination on the dorsal side of the medulla oblongata, appearing as a contrast-enhanced region (arrow). (D) The patient had slight periventricular white matter abnormalities on fluid-attenuated inversion recovery (FLAIR) magnetic resonance imaging. (E) Contrast-enhanced T1-weighted magnetic resonance imaging 2 months after treatment demonstrated a complete response. (F) The patient clearly showed exacerbated periventricular white matter abnormalities on FLAIR imaging and gradually developed a neurocognitive disorder in the 6 years following treatment.

As regards treatment selection and outcome, the two patients who received craniospinal and whole-ventricular irradiation after biopsy-only surgery exhibited a long-term survival of $\geq 6$ years (Table II, cases 3 and 4). However, two patients experienced relapses consisting of cerebrospinal dissemination after treatment and eventually succumbed to the disease (Table II, cases 1 and 4). Of the two patients with recurrence, one had only received local irradiation at the site of the pineal tumor without chemotherapy after R2-resection. These outcomes suggest that the irradiation field may affect recurrence, although we cannot clearly establish an association between the irradiation field and the outcome, due to the limited number of cases.

Toxicity. All the patients recovered from surgery without significant problems. No lethal events or serious intracranial bleeding were recorded in the perioperative period.

Table II outlines the late adverse effects after treatment. The two patients who received 36 Gy of craniospinal irradiation and 18 Gy of whole-ventricular irradiation had evidence of cerebral white matter abnormalities in MRI and grade 3 cognitive disturbance according to RTOG and CTCAE toxicity grading, at 5-7 months and at 4-6 years after radiation therapy (Table II, Fig. 1). Although the patient who received only 34.2 Gy of whole-ventricular irradiation had evidence of cerebral white matter abnormalities at 6 months after radiation therapy, the patient exhibited no evidence of a neurocognitive disorder, even 9 years after the treatment. The patients who were irradiated locally had no evidence of cerebral white matter abnormalities or neurocognitive disorders. The two patients who received craniospinal and whole-ventricular irradiation also exhibited evidence of hypopituitarism after treatment: one developed a growth hormone deficiency 8 months after radiation therapy and the other developed a thyroid-stimulating hormone deficiency 7 years after radiation therapy. However, the latter patient already had a lack of growth hormone when he was diagnosed with PPTID. There were no reported cerebrovascular disorders after treatment.

\section{Discussion}

As the number of available studies on the treatment of PPTID is limited, the present study may add noteworthy evidence regarding viable treatment outcomes with radiation therapy and chemotherapy in PPTID. In our cohort, two patients experienced relapses with cerebrospinal dissemination and eventually succumbed to the disease; this was consistent with previous studies suggesting the potentially aggressive behavior of PPTID and its tendency for CSF seeding $(8,11)$. In a previous report analyzing patterns of prognostic factors and treatment failures, five of 37 patients with PPTID relapsed $>5$ years after the initial treatment (11). The role of craniospinal and whole-ventricular irradiation for patients with PPTID remains to be determined. However, the importance of irradiation for patients with pineal parenchymal tumors, excluding pineocytoma, was investigated in a previous study on a series of 30 patients with pineal tumors and 105 with other germ cell tumors (12). That study demonstrated an association between the radiation dose administered and survival time in patients with pineal parenchymal tumors: the patients who received doses $>50$ Gy had a significantly higher 3 -year survival rate compared to those who received lower doses ( 94 vs. $56 \%$, respectively; $\mathrm{P}=0.03$ ). In the present study, almost all the patients, even those with cerebrospinal dissemination 
at diagnosis, survived long-term after radiation therapy (Table II). In our cohort, one of the two patients diagnosed with cerebrospinal dissemination received a total of 54 Gy of craniospinal and whole-ventricular irradiation with a combination of sequential chemotherapy after biopsy-only surgery and survived for $>7$ years. Doses $>50 \mathrm{~Gy}$ and wide irradiation fields, such as craniospinal and whole-ventricular irradiation, combined with sequential chemotherapy, may be an effective treatment for PPTID with dissemination, although the number of cases analyzed was limited.

Whether all PPTID patients should be treated with chemotherapy remains controversial. Among the five PPTID patients in the present study, one did not receive chemotherapy; this patient developed spinal seeding after treatment and succumbed to the disease. In this patient, a radiation dose of $54 \mathrm{~Gy}$ was directed at the pineal region. Given the CSF seeding potential of PPTID and the outcome of this case, either systemic chemotherapy or a wider irradiation field may be required to prevent recurrence, although a definitive conclusion cannot be reached from only five cases regarding the exact indication of chemotherapy and the width of the irradiation field.

There is currrently no standard systemic therapy for PPTID patients. In this study, we used a combination regimen of vincristine, nimustine, carboplatin and interferon $\beta$ as a systemic treatment for PPTID. The safety of the regimen used in this study was previously confirmed by a phase II study in patients with glioblastoma multiforme (13). Previous studies demonstrated promising results with the combination of vincristine, nitrosoureas and platinums in children diagnosed with primitive neuroectodermal tumors and low-grade gliomas $(14,15)$. Interferon $\beta$ was reported to act as a drug sensitizer for nitrosourea and alkylating agents, whereas interferon $\beta$ and nitrosourea combination therapy has been used for the treatment of gliomas in Japan (16). Moreover, interferon $\beta$ was shown to enhance chemosensitivity to alkylating agents by downregulating the expression of a DNA repair protein, $\mathrm{O}^{6}$-methylguanine DNA methyltransferase, via p53 induction (17).

A previous study used combination chemotherapy with cisplatin and vinblastine as systemic treatment of pineal parenchymal cell tumors (18), whereas a clinical study on childhood pineoblastoma used a regimen including ifosfamide, etoposide, high-dose methotrexate, cisplatin and cytarabin followed by radiation therapy, or a regimen including vincristine, lomustine and carboplatin (19). These regimens may represent other chemotherapeutic options for pineal parenchymal tumors. A recent study suggested that molecular-targeted therapies, in addition to chemotherapy, may be a viable treatment option for PPTID tumors. A mutation of epidermal growth factor receptor (in-frame deletion of exons 2-7) was detected in PPTID tumors by flow cytometry, western blot analysis and reverse-transcription polymerase chain reaction (20). Patients with PPTID may benefit from a combination of chemotherapy and molecular-targeted therapy in the future.

Although almost all the patients in the present study were long-time survivors, they experienced some late adverse effects. The two patients who received craniospinal irradiation exhibited severe cognitive impairment (grade 3, RTOG and CTCAE toxicity grading) 4-6 years after radiation therapy. This result is consistent with previous reports regarding neurobehavioral outcomes following cranial irradiation (21). Neurocognitive disorder due to treatment is a multifactorial consequence, although data suggest that injury to neural progenitor cells plays an important role in treament-related neurocognitive toxicity $(22,23)$. Associations between radiation dose to neural progenitor cell niches and the temporal lobes were previously reported (24). From this viewpoint, radiation delivery technique modifications, including hippocampal avoidance, may carry the potential to mitigate cognitive neurotoxicity by sparing normal neural stem cells, as in the RTOG 0933 trial, which was a phase II trial investigating whether avoiding the hippocampus during whole-brain radiation therapy lowers the incidence of long-term neurocognitive toxicity in the management of brain metastases.

In addition to the neurocognitive toxicity effect of radiation therapy, certain types of chemotherapy potentially contribute to neurocognitive toxicity due to the different sensitivity of normal neural stem cells (25). Newer chemotherapy agents, such as the epidermal growth factor receptor tyrosine kinase inhibitor and the proteasome inhibitor, were reported as potentially neurotoxic, compared to conventional chemotherapeutic agents (25). Moreover, irradiation may enhance chemotherapeutic neurocognitive toxicity. The majority of chemotherapeutic agents do not effectively penetrate the blood brain barrier; however, there is evidence that brain irradiation causes radiation-induced damage to the capillary bed (26). The concurrent or subsequent administration of neurotoxic chemotherapy while the blood brain barrier is disrupted by radiation therapy is likely to result in the sensitization of the brain to chemotherapy (21). As PPTID patients have the possibility of long-term survival even after recurrence, as shown in this study, whether patients should receive systemic chemotherapy and craniospinal and whole-ventricular irradiation should be carefully considered, depending on the patient's pathological characteristics, disease extent and potential to develop severe late adverse effects. As there are several available methods to enhance treatment intensity and reduce the possible effects on long-term toxicity, more detailed prospective future studies with a larger number of cases are required to investigate the optimal irradiation field and chemotherapeutic strategy for PPTID.

There were potential limitations to this study. First, we could not prospectively evaluate the cognitive disorders with a general neurocognitive function assessment, such as the Mini-Mental State Examination, the Montreal Cognitive Assessment, the Hopkins Verbal Learning Test and Trail Making Tests, or a more detailed objective patient-reported quality of life outcome assessment. Second, this was a retrospective study with a limited case series. Although this disease entity is rare, we should prospectively investigate larger cohorts to determine the appropriate treatment options.

In conclusion, we evaluated the outcomes of multimodality treatment for PPTID. Patients with PPTID in this study survived long-term, even after experiencing a recurrence. However, some patients developed serious neurocognitive disorders a few years after the treatment. Taking into account the rarity of this disease and the long-term survival of recurrent patients, a prospective multi-institutional study including a large patient cohort is required to determine the optimal width of the irradiation field and the use of chemotherapy after surgery, weighing the serious late adverse events and survival time. 


\section{Acknowledgements}

This study was supported in part by Grants-in-Aid for scientific research from the Ministry of Education, Culture, Sports, Science and Technology (no. 20229009) of Japan.

\section{References}

1. Schild SE, Scheithauer BW, Schomberg PJ, Hook CC, Kelly PJ, Frick L, Robinow JS and Buskirk SJ: Pineal parenchymal tumors. Clinical, pathologic, and therapeutic aspects. Cancer 72: 870-880, 1993.

2. Jouvet A, Saint-Pierre G, Fauchon F, Privat K, Bouffet E, Ruchoux MM, Chauveinc L and Fèvre-Montange M: Pineal parenchymal tumors: a correlation of histological features with prognosis in 66 cases. Brain Pathol 10: 49-60, 2000.

3. Louis DN, Ohgaki H, Wiestler OD, Cavenee WK, Burger PC, Jouvet A, Scheithauer BW and Kleihues P: The 2007 WHO classification of tumours of the central nervous system. Acta Neuropathol 114: 97-109, 2007.

4. Clark AJ, Ivan ME, Sughrue ME, Yang I, Aranda D, Han SJ, Kane AJ and Parsa AT: Tumor control after surgery and radiotherapy for pineocytoma. J Neurosurg 113: 319-324, 2010.

5. Clark AJ, Sughrue ME, Ivan ME, Aranda D, Rutkowski MJ, Kane AJ, Chang S and Parsa AT: Factors influencing overall survival rates for patients with pineocytoma. J Neurooncol 100: 255-260, 2010 .

6. Fangusaro J, Finlay J, Sposto R, Ji L, Saly M, Zacharoulis S, Asgharzadeh S, Abromowitch M, Olshefski R, Halpern S, Dubowy R, Comito M, Diez B, Kellie S, Hukin J, Rosenblum M, Dunkel I, Miller DC, Allen J and Gardner S: Intensive chemotherapy followed by consolidative myeloablative chemotherapy with autologous hematopoietic cell rescue (AuHCR) in young children with newly diagnosed supratentorial primitive neuroectodermal tumors (sPNETs): report of the Head Start I and II experience. Pediatr Blood Cancer 50: 312-318, 2008.

7. Jouvet A, Fèvre-Montange M, Besançon R, Derrington E, Saint-Pierre G, Belin MF, Pialat J and Lapras C: Structural and ultrastructural characteristics of human pineal gland, and pineal parenchymal tumors. Acta Neuropathol 88: 334-348, 1994.

8. Fauchon F, Jouvet A, Paquis P, Saint-Pierre G, Mottolese C, Ben Hassel M, Chauveinc L, Sichez JP, Philippon J, Schlienger M and Bouffet E: Parenchymal pineal tumors: a clinicopathological study of 76 cases. Int J Radiat Oncol Biol Phys 46: 959-968, 2000.

9. Maarouf M, El Majdoub F, Bührle C, Voges J, Lehrke R, Kocher M, Hunsche S, Treuer H and Sturm V: Pineal parenchymal tumors. Management with interstitial iodine-125 radiosurgery. Strahlenther Onkol 186: 127-134, 2010.

10. Stoiber EM, Schaible B, Herfarth K, Schulz-Ertner D, Huber PE, Debus J and Oertel S: Long term outcome of adolescent and adult patients with pineal parenchymal tumors treated with fractionated radiotherapy between 1982 and 2003 - a single institution's experience. Radiat Oncol 5: 122, 2010.

11. Lutterbach J, Fauchon F, Schild SE, Chang SM, Pagenstecher A, Volk B, Ostertag C, Momm F and Jouvet A: Malignant pineal parenchymal tumors in adult patients: patterns of care and prognostic factors. Neurosurgery 51: 44-55, 2002.

12. Schild SE, Scheithauer BW, Haddock MG, Wong WW, Lyons MK, Marks LB, Norman MG and Burger PC: Histologically confirmed pineal tumors and other germ cell tumors of the brain. Cancer 78 : 2564-2571, 1996.
13. Aoki T, Takahashi JA, Ueba T, Oya N, Hiraoka M, Matsui K, Fukui T, Nakashima Y, Ishikawa M and Hashimoto N: Phase II study of nimustine, carboplatin, vincristine, and interferon- $\beta$ with radiotherapy for glioblastoma multiforme: experience of the Kyoto Neuro-Oncology Group. J Neurosurg 105: 385-391, 2006.

14. Packer RJ, Lange B, Ater J, Nicholson HS, Allen J, Walker R, Prados M, Jakacki R, Reaman G and Needles MN: Carboplatin and vincristine for recurrent and newly diagnosed low-grade gliomas of childhood. J Clin Oncol 11: 850-856, 1993.

15. Packer RJ, Sutton LN, Elterman R, et al: Outcome for children with medulloblastoma treated with radiation and cisplatin, CCNU, and vincristine chemotherapy. J Neurosurg 81: 690-698, 1994.

16. Wakabayashi T, Hatano N, Kajita Y, Yoshida T, Mizuno M, Taniguchi K, Ohno T, Nagasaka T and Yoshida J: Initial and maintenance combination treatment with interferon-beta, MCNU (ranimustine), and radiotherapy for patients with previously untreated malignant glioma. J Neurooncol 49: 57-62, 2000.

17. Natsume A, Ishii D, Wakabayashi T, Tsuno T, Hatano $H$, Mizuno $M$ and Yoshida J. IFN-beta down-regulates the expression of DNA repair gene MGMT and sensitizes resistant glioma cells to temozolomide: Cancer Res 65: 7573-7579, 2005.

18. Kurisaka M, Arisawa M, Mori T, Sakamoto T, Seike M, Mori K, Okada T, Wakiguchi $\mathrm{H}$ and Kurashige T: Combination chemotherapy (cisplatin, vinblastin) and low-dose irradiation in the treatment of pineal parenchymal cell tumors. Childs Nerv Syst 14: 564-569, 1998.

19. Hinkes BG, von Hoff K, Deinlein F, Warmuth-Metz M, Soerensen N, Timmermann B, Mittler U, Urban C, Bode U, Pietsch T, Schlegel PG, Kortmann RD, Kuehl J and Rutkowski S: Childhood pineoblastoma: experiences from the prospective multicenter trials HIT-SKK87, HIT-SKK92 and HIT91. J Neurooncol 81: 217-223, 2007.

20. Li G, Mitra S, Karamchandani J, Edwards MS and Wong AJ: Pineal parenchymal tumor of intermediate differentiation: clinicopathological report and analysis of epidermal growth factor receptor variant III expression. Neurosurgery 66: 963-968, 2010

21. Crossen JR, Garwood D, Glatstein E and Neuwelt EA: Neurobehavioral sequelae of cranial irradiation in adults: a review of radiation-induced encephalopathy. J Clin Oncol 12: 627-642, 1994

22. Monje ML, Mizumatsu S, Fike JR and Palmer TD: Irradiation induces neural precursor-cell dysfunction. Nat Med 8: 955-962, 2002.

23. Mizumatsu S, Monje ML, Morhardt DR, Rola R, Palmer TD and Fike JR: Extreme sensitivity of adult neurogenesis to low doses of X-irradiation. Cancer Res 63: 4021-4027, 2003.

24. Redmond KJ, Mahone EM, Terezakis S, Ishaq O, Ford E, McNutt T, Kleinberg L, Cohen KJ, Wharam M and Horska A: Association between radiation dose to neuronal progenitor cell niches and temporal lobes and performance on neuropsychological testing in children: a prospective study. Neuro Oncol 15: 360-369, 2013.

25. Gong X, Schwartz PH, Linskey ME and Bota DA: Neural stem/progenitors and glioma stem-like cells have differential sensitivity to chemotherapy. Neurology 76: 1126-1134, 2011.

26. Brown WR, Blair RM, Moody DM, Thore CR, Ahmed S, Robbins ME and Wheeler KT: Capillary loss precedes the cognitive impairment induced by fractionated whole-brain irradiation: a potential rat model of vascular dementia. J Neurol Sci 257: 67-71, 2007. 